\title{
Mineração
}

\section{Programa computacional para simulação do rendimento de ejetores para fins de dragagem}

\author{
Iran E. Lima Neto \\ Doutorando em Recursos Hídricos (bolsista CAPES) \\ Universidade de Alberta, Edmonton, $A B$, Canadá. \\ E-mail:limaneto@ualberta.ca
}

\section{Resumo}

Desenvolveu-se, nessa pesquisa, um programa computacional para previsão e análise do rendimento de ejetores para bombeamento de misturas de água com sólidos em suspensão, nas atividades de mineração. A estrutura do programa consiste na entrada de dados de curvas características obtidas com o ejetor operando com água, tanto como fluido motriz, como de sucção, fornecidas pelo fabricante, para calibração dos coeficientes de perda de carga e simulação do rendimento de tal equipamento para diferentes vazões, massas específicas e concentrações de sólidos no fluido de sucção, baseando-se nas equações de conservação de massa, quantidade de movimento e energia. Os resultados obtidos com o programa apresentaram adequada concordância com dados experimentais disponíveis na literatura.

Palavras-chave: Programa computacional, ejetor, rendimento, dragagem .

\begin{abstract}
A computer program was developed in this research to predict and evaluate the performance of ejectors for pumping of mixtures of water and solids in suspension in the mining activities. The structure of the program consists in inputting data points from characteristic curves obtained with the ejector operating with water both as driving and suction fluid, supplied by the manufacturer, for calibration of the head loss coefficients and simulation of the ejector performance for different flowrates, specific mass and concentration by weight of the solids in the suction fluid, based on the conservation equations for mass, momentum and energy. The results obtained with the program presented good agreement with experimental data available in the literature.
\end{abstract}

Keywords: Computer program, ejector, efficiency, dredging. 


\section{Introdução}

O estudo de bombeamento de misturas de água com sólidos em suspensão consiste em uma das principais tarefas dos engenheiros e demais profissionais que atuam nas áreas de mineração, uma vez que o sistema de bombeamento selecionado será responsável pela dragagem do minério presente na jazida e pelo seu envio às instalações de beneficiamento. Como, nessa etapa de bombeamento, os sólidos em suspensão geralmente apresentam granulometria maior que a da areia, as bombas centrífugas utilizadas para cumprir tal papel normalmente têm seu rotor obstruído ou erodido após um curto período de uso, o que acarreta um maior tempo de parada do sistema e, conseqüentemente, em maiores custos de produção do minério [3].

Nesse contexto, o uso de ejetores para bombeamento de misturas águasólidos em suspensão surge como uma alternativa bastante atrativa, visto que esses equipamentos de baixo custo não apresentam partes móveis (rotores, êmbolos, etc), descartando-se, assim, a freqüente necessidade de manutenção do sistema, como no caso das bombas centrífugas. Geralmente fabricados com chapas e tubos metálicos modelados em torno, os ejetores são compostos basicamente por cinco partes: bocais de alimentação e de sucção, câmara de sucção, câmara de mistura, ou garganta, e difusor, conforme representado na Figura 1.

Utilizando a energia hidráulica normalmente proveniente de uma bomba centrífuga, os ejetores são usados para aspirar e recalcar um fluido qualquer através da criação de um vácuo na câmara de sucção (Figura 2). Isto ocorre porque o jato na saída do bocal de alimentação converte o trabalho de pressão em energia cinética e, conseqüentemente, a pressão nesse ponto torna-se menor do que a atmosférica, provocando o arraste do fluido de sucção. Em seguida, ocorre mistura turbulenta na garganta até que $o$ fluido combinado entra no difusor e, na medida em que a velocidade vai diminuindo, há um ganho de pressão. Devido às perdas produzidas, a pressão de saída do aparelho é sempre inferior à de entrada.
Diversas pesquisas têm sido desenvolvidas no sentido de se obterem soluções analíticas ou métodos numéricos para simulação do rendimento de ejetores operando com água, tanto como fluido motriz, como de sucção ([2], [4], [5], [7]). No entanto, pouco avanço científico tem sido observado no estudo de ejetores para transporte de material particulado, no qual geralmente assumem-se parâmetros empíricos para simulação do rendimento desses equipamentos [6]. Com o objetivo de contribuir para o estudo de dragagem nas atividades de mineração, essa pesquisa apresenta um programa computacional que se utiliza apenas de informações básicas como dados de curvas características fornecidas pelo fabricante do ejetor, diâmetros de saída do bocal de alimentação e da garganta, peso específico do sólido a ser bombeado e sua concentração no fluido de sucção para simulação do rendimento de tal aparelho.

\section{Equacionamento Proposto}

Através de um balanço de potência entre as seções $4 a, 4 b$ e $4 c$ (Figura 2), obtém-se uma equação representativa do rendimento do ejetor expressa em termos da relação de vazão entre os fluidos de sucção e motriz $(M)$ e da relação de carga $(N)$ :

$$
\eta=M N=\frac{Q_{3 b}}{Q_{3 a}} \cdot \frac{H_{4 c}-H_{4 b}}{H_{4 a}-H_{4 c}}
$$

Logo, aplicando as equações de continuidade e energia através das seções 4a, 4b e 4c (Figura 2) e a equação de quantidade de movimento entre as se-

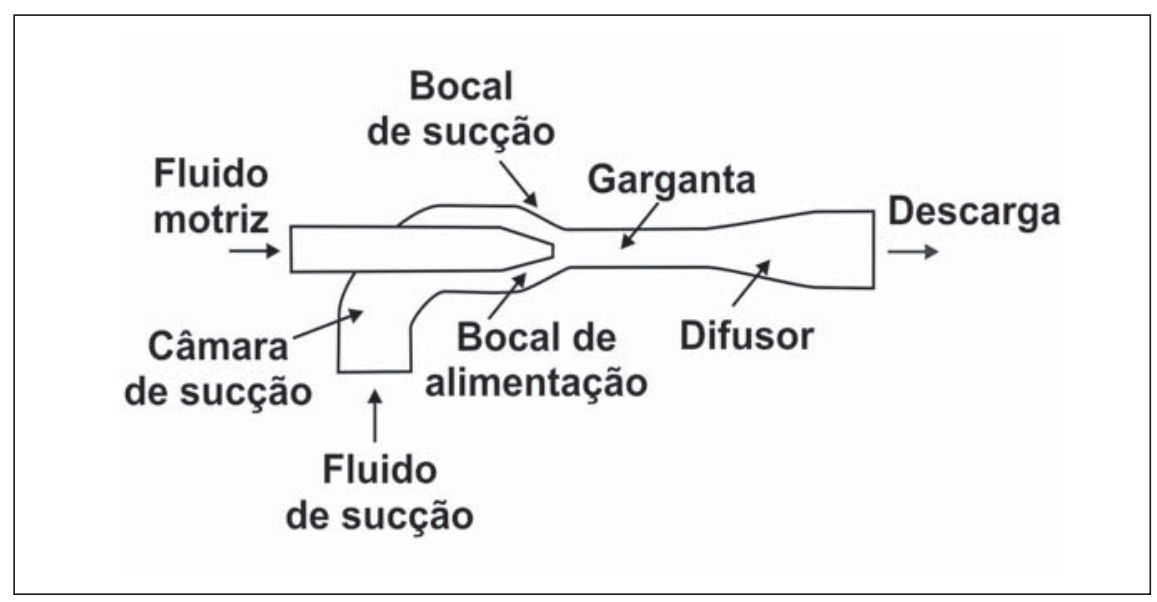

Figura 1 - Componentes de um ejetor.

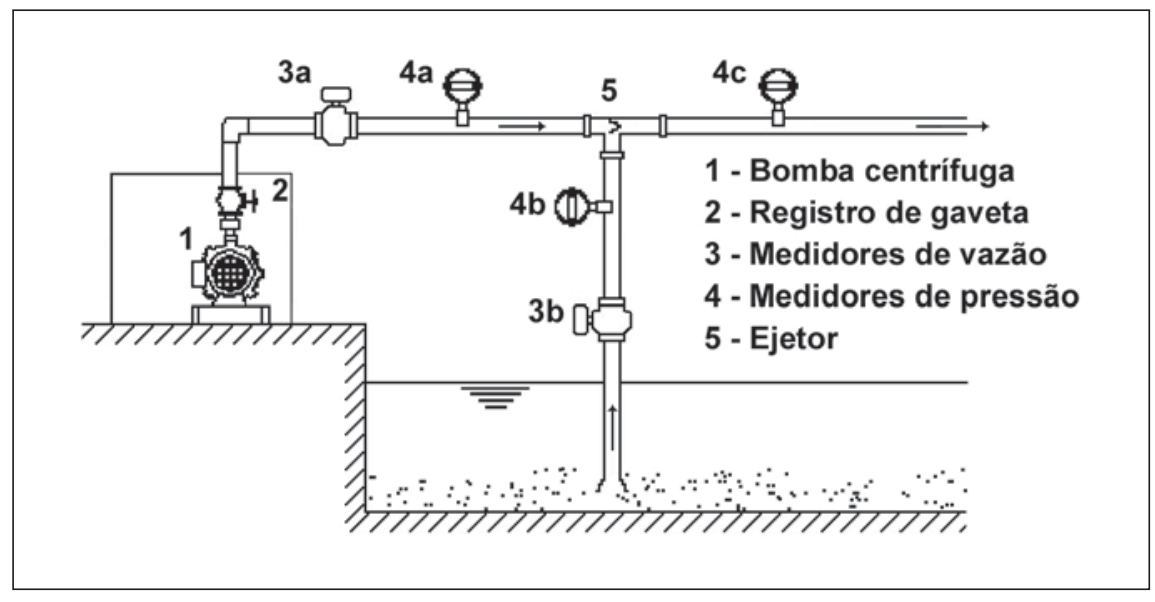

Figura 2 - Esquema de instalação de um sistema bomba centrífuga-ejetor. 
ções de entrada e saída da garganta (Figura 1), obtém-se uma fórmula teórica para o rendimento em função de $M$ e $N^{\prime}$, onde $N^{\prime}$ é a relação de carga teórica expressa em termos de $M$, da relação entre as áreas do jato e da garganta do ejetor $(R)$, do peso específico do fluido de sucção $(S)$ e dos coeficientes de perda de carga no bocal de alimentação $\left(K_{B}\right)$, na câmara de sucção $\left(K_{S}\right)$, na garganta $\left(K_{G}\right)$ e no difusor $\left(K_{D}\right)$ :

$$
N^{\prime}=\frac{2 R+S\left[\frac{M^{2} R^{2}}{1-R}\left(2-\frac{1+S K_{S}}{1-R}\right)\right]-(1+S M)\left\{R^{2}(1+M)\left[1+\frac{1+S M}{1+M}\left(K_{G}+K_{D}\right)\right]\right\}}{1+K_{B}-2 R-S\left[\frac{2 M^{2} R^{2}}{1-R}\right]+(1+S M)\left\{R^{2}(1+M)\left[1+\frac{1+S M}{1+M}\left(K_{G}+K_{D}\right)\right]\right\}}
$$

em que $S$ é expresso em termos do peso específico do sólido a ser bombeado $\left(\gamma_{S}\right)$ e da sua concentração em peso no fluido de sucção $\left(C_{S}\right)$ :

$S=C_{S}\left(\gamma_{S}-1\right)$

$\mathrm{Na}$ derivação da Eq. (2), considerou-se que a perda de carga em cada componente do ejetor para bombeamento de misturas água-sólidos em suspensão é proporcional a sua respectiva perda de carga para bombeamento de água pura multiplicada pelo peso específico da mistura [1]. Logo, os coeficientes de perda de carga no bocal de alimentação, na câmara de sucção, na garganta e no difusor foram expressos respectivamente por $K_{B}, S K_{S},(1+S M) /(1+M) K_{G}$ e $(1+S M) /(1+M) K_{D}$.

Portanto, substituindo-se $N$ por $N$ ' na Eq.(1), o rendimento teórico é expresso por:

$\eta^{\prime}=M N^{\prime}$

\section{Programa Computacional}

Desenvolveu-se, nessa pesquisa, o programa computacional SRED (Simulação do Rendimento de Ejetores para fins de Dragagem) em ambiente Matlab, o qual utiliza os seguintes dados de entrada: relação de áreas $R$ e pontos da curva característica do ejetor operando com água pura como fluidos motriz e de sucção para calibração dos coeficientes de perda de carga $K_{B}, K_{S}$, $K_{G}$ e $K_{D}$, utilizando ferramentas de otimização do Matlab para minimização do desvio médio quadrático entre os dados teóricos (Eq. 4 para $S=1$ ) e experimentais (curva característica fornecida pelo fabricante do equipamento); e peso específico do sólido a ser bombeado $\gamma_{s}$, sua concentração em peso no fluido de sucção $C_{S}$ e relação de vazão $M$ máxima desejada para simulação do rendimento do ejetor (Eq. 4 para $S>1$ ). O programa também apresenta a opção de entrada direta dos coeficientes de perda de carga para simulação do rendimento do ejetor ("simulação direta"), caso o usuario já tenha realizado o processo de calibração. O fluxograma básico do referido programa é apresentado na Figura 3.

Na calibração dos coeficientes de perda de carga $K_{B}, K_{S}, K_{G}$ e $K_{D}$ através do método de mínimos quadrados foram adotadas restrições de acordo com os valores limites de cada um desses coeficiente obtidos na literatura [5], [7].

\section{Resultados e discussão}

$\mathrm{Na}$ Figura 4, é apresentado o ajuste das curvas teóricas de rendimento (simulação com o programa SRED para $S=1$ ) aos dados experimentais (curva característica para água como fluidos motriz e de sucção obtida por Prabkeao e Aoki [6]) para calibração dos coeficientes de perda de carga em cada componente de ejetores com relações de áreas $R$ de 0,09; 0,16 e 0,36.

A calibração forneceu os seguintes coeficientes de perda de carga no bocal de alimentação $\left(K_{B}\right)$, na câmara de sucção $\left(K_{S}\right)$, na garganta $\left(K_{G}\right)$ e no difusor $\left(K_{D}\right)$, e coeficientes de correlação $\left(C_{C}\right)$ entre as curvas teóricas e os dados experimentais para cada ejetor (Tabela 1).

Os coeficientes de correlação obtidos mostraram excelente ajuste das curvas teóricas aos dados experimentais, o que significa que os valores dos coeficientes de perda de carga calibrados são coerentes e similares àqueles obtidos na literatura [5], [7].

Na Figura 5, é apresentado o ajuste das curvas teóricas de rendimento (simulação com o programa SRED para $S=2,07$ ) aos dados experimentais (curva característica para água como fluido motriz e mistura de água com $65 \%$ de sólidos de massa específica de $2650 \mathrm{~kg} / \mathrm{m}^{3}$ como fluido de sucção obtida por Prabkeao e Aoki [6]) para os ejetores com relações de áreas $R$ de 0,09 ; $0,16 \mathrm{e} 0,36$. 
Os coeficientes de correlação $C_{C}$, para os ejetores com valores de $R$ iguais a 0,$09 ; 0,16$ e 0,36 , foram, respectivamente, 0,$9955 ; 0,9900$ e 0,9308 , o que indica que o programa SRED apresentou bom ajuste das curvas teóricas aos dados experimentais. Embora os ejetores com relações de áreas de 0,09 e 0,16 não tenham atingido experimentalmente os seus rendimentos máximos, os resultados obtidos com o programa mostraram que o rendimento máximo desses equipamentos é reduzido em torno de $46 \%$, bem como as relações de vazão $M$, para as quais esses rendimentos ocorrem, quando se utiliza a mistura de água com sólidos em suspensão $(S=2,07)$, ao invés de água pura $(S=1,0)$ como fluido de sucção.

\section{Conclusões}

O programa computacional proposto nessa pesquisa para avaliar o rendimento de ejetores para fins de dragagem apresentou valores adequados para os coeficientes de perda de carga calibrados e bons ajustes aos dados experimentais disponíveis na literatura. Os resultados permitiram evidenciar que, quanto maior o peso específico do sólido a ser bombeado e/ou a concentração do mesmo no fluido de sucção, menores são os faixas de vazão de operação e os rendimentos alcançados.

Sugere-se, como futuras pesquisas, a análise do programa para simulação do rendimento de ejetores operando com diferentes pesos específicos e concentrações de sólidos no fluido de sucção, bem como a verificação do efeito da granulometria dos sólidos a serem bombeados na eficiência de tais equipamentos.

\section{Agradecimentos}

O autor agradece ao Prof. Dr. Rodrigo de Melo Porto, orientador da pesquisa de mestrado de onde foram obtidas importantes informações para elaboração deste artigo, e à Coordenadoria de Aperfeiçoamento de Pessoal de Nível Superior - CAPES pela bolsa de estudos concedida.

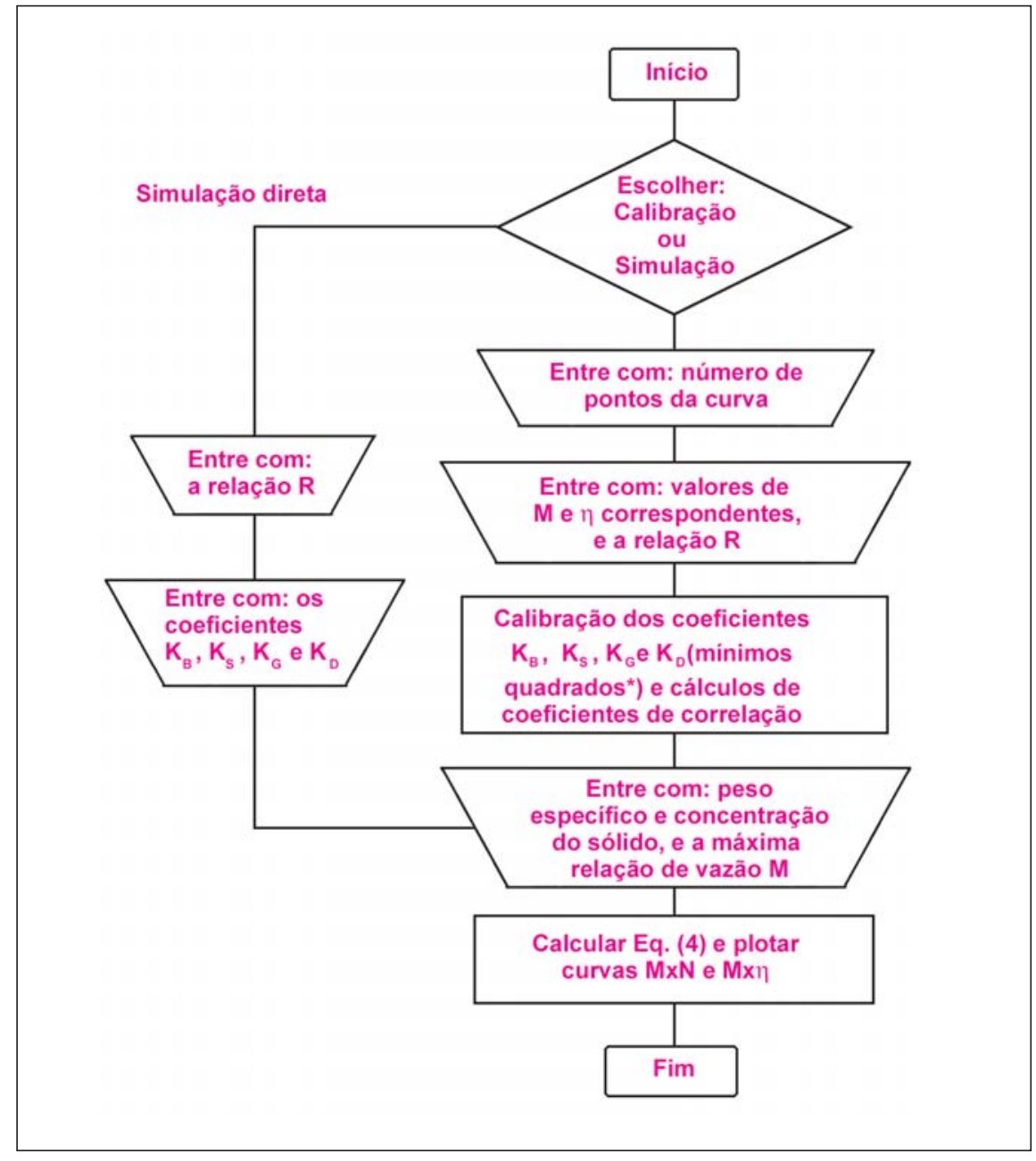

Figura 3 - Fluxograma do Programa SRED.

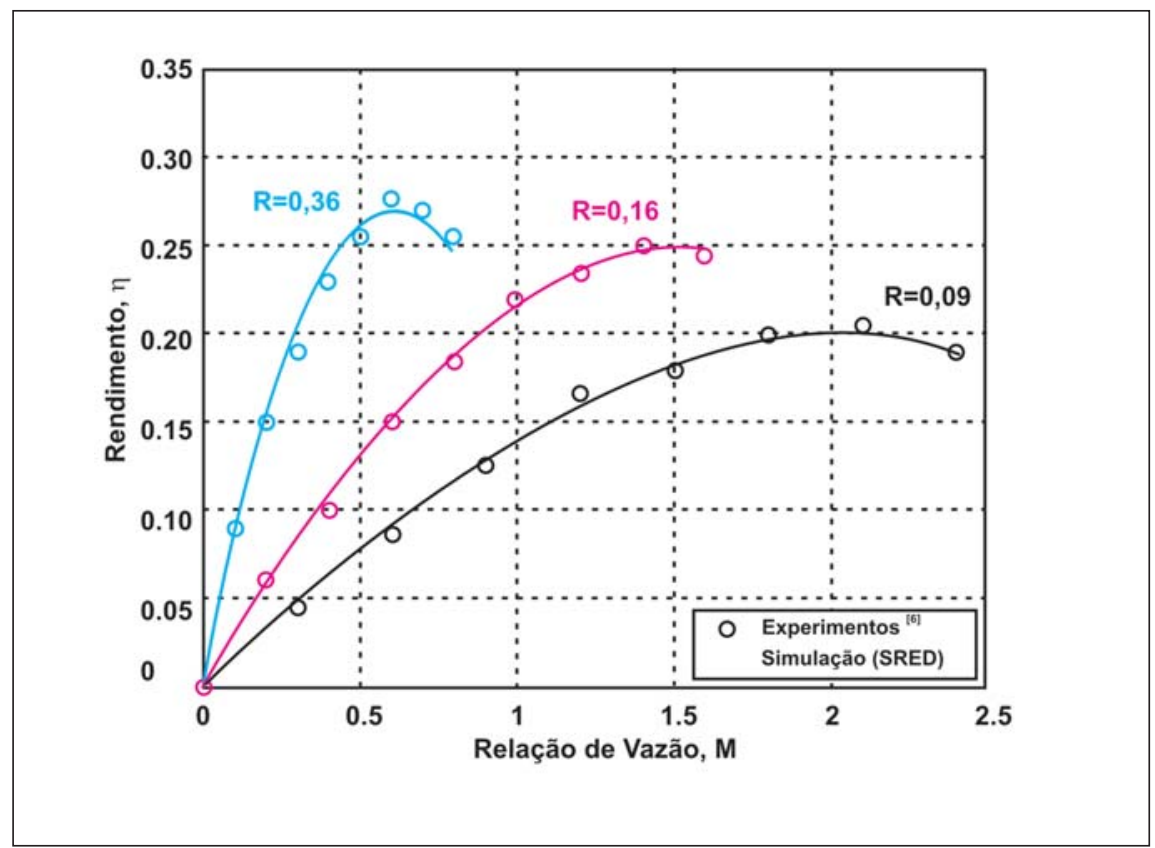

Figura 4 - Ajuste das curvas teóricas ( $\eta$ ') aos dados experimentais $(\eta)$ para $S=1,0$ (Calibração). 
Iran E. Lima Neto

Tabela 1 - Resultados da calibração.

\begin{tabular}{c|c|c|c|c|c}
\hline $\boldsymbol{R}$ & $\boldsymbol{K}_{\boldsymbol{B}}$ & $\boldsymbol{K}_{\boldsymbol{S}}$ & $\boldsymbol{K}_{\boldsymbol{G}}$ & $\boldsymbol{K}_{\boldsymbol{D}}$ & $\boldsymbol{C}_{\boldsymbol{C}}$ \\
\hline 0,09 & 0,1938 & 0,6425 & 0,0500 & 0,1010 & 0,9987 \\
\hline 0,16 & 0,2424 & 0,4003 & 0,0500 & 0,1000 & 0,9990 \\
\hline 0,36 & 0,2005 & 0,4000 & 0,0500 & 0,1000 & 0,9966 \\
\hline
\end{tabular}

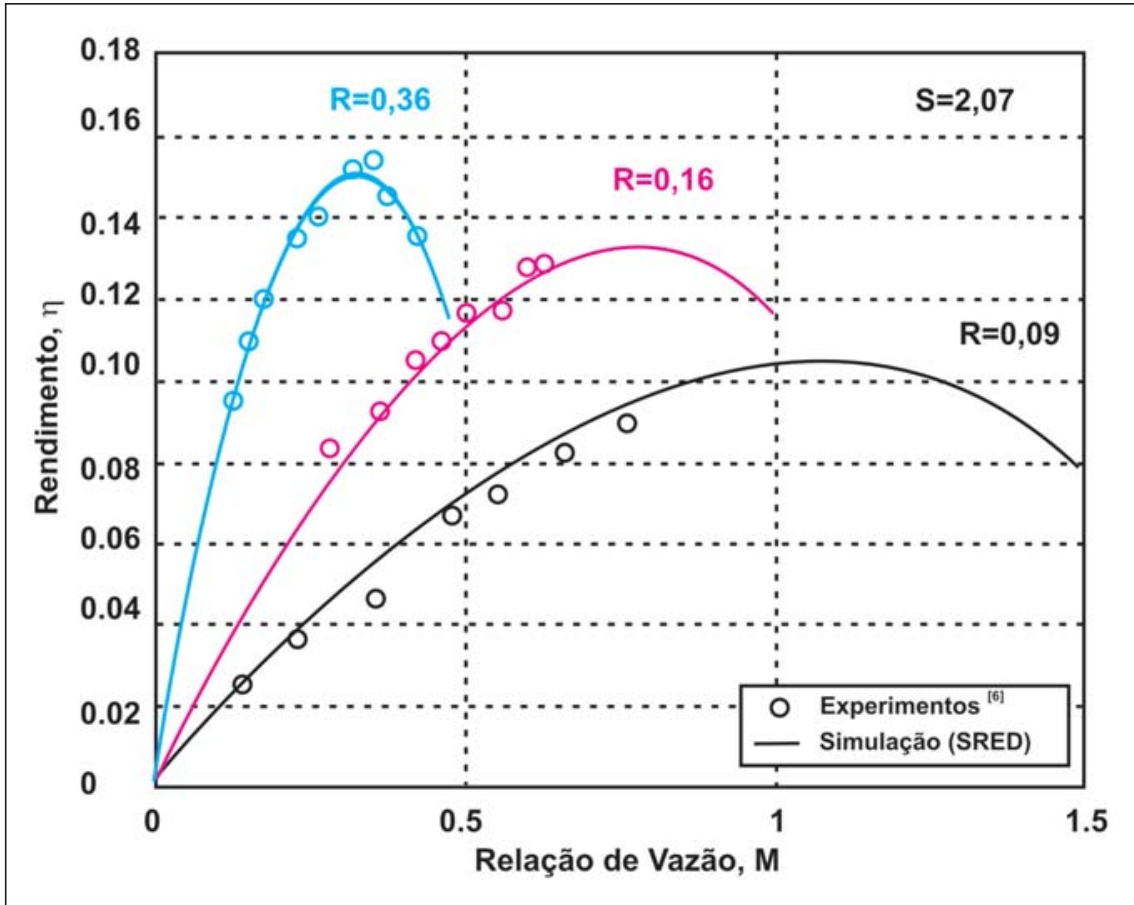

Figura 5 - Ajuste das curvas teóricas ( $\eta$ ') aos dados experimentais $(\eta)$ para $S=2,07$.

\section{Informações adicionais}

O programa SRED pode ser obtido através de contato direto com o endereço eletrônico do autor.

\section{Referências bibliográficas}

[1] COULSON, J. M., RICHARDSON, J. R., BACKHURST, J. H., HARKER, J. H. Fluid flow heat transfer and mass transfer, butterworth and heinemann. In: Chemical Engineering. v.1, 1996, 895p.

[2] HONGJI, L., XIANGJIN, Z. Studies on liquid jet pump. Scientia Sinica, v.24, n.4, p.581593, 1981.

[3] LIMA NETO, I. E. Desenvolvimento $\boldsymbol{e}$ avaliação experimental de ejetores de baixo custo. Escola de Engenharia de São Carlos, Universidade de São Paulo, 2001. (Dissertação de Mestrado).

[4] Lima NETO, I. E., PORTO, R. M. Performance of low-cost ejectors. Journal of Irrigation and Drainage Engineering, ASCE, v.130, n.2, p.122-128, 2004.

[5] MUELleR, N. H. G. Water jet pump. Journal of the Hydraulics Division, ASCE, v.90, n.3, p.83-113, 1964.

[6] PRABKEAO, C., AOKI, K. Theoretical analysis on hydraulic transportation of solids using the jet pump. Proceedings of the School of Engineering, Tokai University, v.26, p.1-7, 2001.

[7] REDDY, Y. R., KAR, S. Theory and performance of water jet pump. Journal of the Hydraulics Division, ASCE, v.94, n.5, p.1261-1281, 1968.

Artigo recebido em 05/08/2004 e aprovado em 14/09/2004.

$* * * * * *$

\section{REM - Revista Escola de Minas 68 anos divulgando CIÊNCIA.}

$* * * * * *$

www.rem.com.br

$* * * * * *$ 